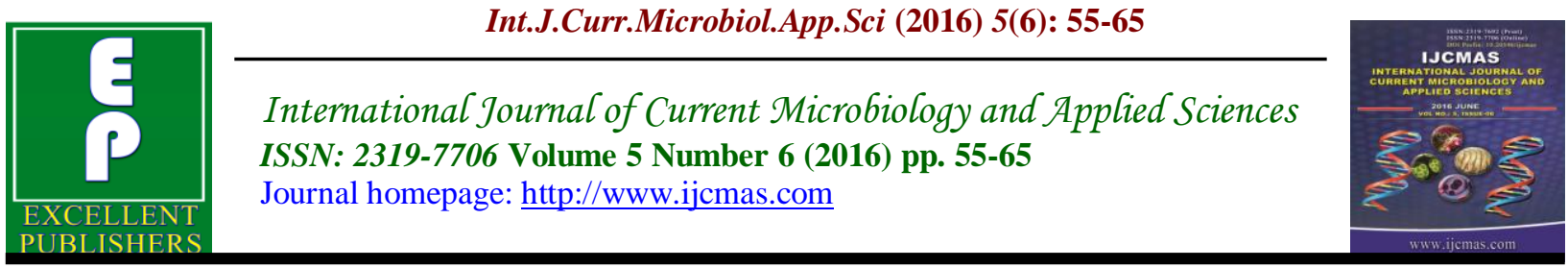

Original Research Article

http://dx.doi.org/10.20546/ijcmas.2016.506.007

\title{
Study of Risk Factors Associated with Candidiasis and Identification and Antifungal Susceptibility Pattern of Candida Isolates in a Tertiary Care Hospital
}

\author{
Shilpa Rajesh Shah* and Swati Raghunath Sapkal \\ Department of Microbiology, Bharati Vidyapeeth Deemed University Medical \\ College and Hospital, Sangli-416410, India
}

*Corresponding author

\section{A B S T R A C T}

\begin{tabular}{l} 
K e y w o r d s \\
Candida albicans, \\
Non albicans \\
candida (NAC), \\
CHROM agar, \\
antifungal \\
susceptibility \\
\hline Article Info \\
\hline Accepted: \\
07 May 2016 \\
Available Online: \\
10 June 2016
\end{tabular}

The incidence of serious fungal infections continues to rise. Amongst them infections due to candida species are increasing in the last few decades due to increase in HIV infection, immune compromised conditions like use of steroids, broad spectrum antibiotics, drug abuse, organ transplantation\& so on. The distribution of Candida species causing infections is changing. Till recent past, $C$. albicans was the most common species causing infections but more recently the infections caused due to non albicans candida (NAC) species are on rise. The present study was planned for studying the various clinical isolates of candida, its predisposing factors, the use of CHROM agar for rapid identification and its antifungal susceptibility pattern. A total of 100 clinical isolates of candida reported as pathogenic were studied for identification by conventional method and by growth on CHROM agar and corn meal agar. It's antifungal susceptibility testing was done on Muller Hinton with glucose and methylene blue agar by disc diffusion method. The clinical history of the patients was elicitated for risk factors. Out of 100 candida isolates, 41 strains were C. albicans and 59 were NAC belonging to different species, commonest being $C$. tropicalis. Maximum isolates were obtained from urine sample and Diabetes mellitus was found to be the commonest risk factor associated (34\%) followed by pregnancy (16\%). The C. albicans showed highest sensitivity to Amphotericin B followed by Fluconazole. NAC showed maximum sensitivity to Voriconazole followed by Fluconazole and Amphotericin B. Rapid identification of different candida species is possible by growth on CHROM agar by differences in the color of the colonies and the study of morphology on corn meal agar. It is important to do species identification and antifungal susceptibility, as susceptibility varies according to the type of infecting candida species and hence use of CHROM agar with disc diffusion susceptibility testing on methylene blue glucose Muller Hinton agar is a simple, cost effective method. It can be used in resource restricted settings without requirement of any costly instrument. 


\section{Introduction}

The incidence of serious fungal infections continues to rise (Price et al., 1994). Infections due to Candida species are increasing in the last few decades. The most probable reasons being the increasing incidence of HIV infection worldwide and other immunocompromised conditions like use of steroids and broad spectrum antibiotics, drug abuse, organ transplantation, long term hospitalization, intravascular catheters and underlying diseases like diabetes and malignancy (Kashid et al., 2011). The genus Candida comprises about 200 species of which close to 20 have been associated with pathology in humans or animals (Charlene et al., $10^{\text {th }}$ ED).

The yeast C.albicans commonly inhabits in oral and vaginal mucosa and gastrointestinal tract of human being as one of the commensal organisms (Bhavan, 2010, Enoch, 2006). It causes opportunistic infections in immunocompromised patients, produces allergic reactions and rarely causes morbidity and mortality. It also causes life threatening disseminated candidiasis (Bhavan, 2010).

Candida species have been reported as seventh most common nosocomial pathogen (Charlene, et al., 10 ${ }^{\text {th }}$ ED). Epidemiological data from the Indian subcontinent showed that 67 to $90 \%$ of nosocomial candidaemia cases were due to non albicans candida (NAC) species of which Candida tropicalis was the most predominant (Kothavade et al., 2011). The extensive use of antifungal for prophylaxis in these patients became the leading cause of colonization of non albicans candida (NAC) species and increasing resistance to antifungal drugs (Kothavade et al., 2011).

Recently, the incidence of life threatening fungal infections has been on the rise and rapid identification of pathogenic yeasts and detection of polyfungal infections has become mandatory. Chromogenic media can help to reduce the time of isolation and identification as well as detection of mixed cultures by 48-72 hours (Chaudhary et al., 2009). Accurate species identification is important for the treatment of the Candida infections as the non albicans species of Candida continue to be increasingly documented and not all species respond to the same treatment (Dharwad et al., 2011). It has been stated that each species of candida and its susceptibility depends on geographic region, clinical specimen type and hospital location. (Pfaller et al., 2010)

Hence the present study was planned to establish the techniques for rapid identification of Candida up to species level and detecting their antifungal susceptibility pattern and also to study the risk factors involved in candidial infections.

The rapid identification of infecting Candida species and knowledge of their antifungal sensitivity is definitely beneficial to the clinicians for treating Candidial infections.

\section{Materials and Methods}

This study was carried out in Department of Microbiology, Bharati Vidyapeeth Deemed University Medical College and Hospital Sangli, which is a tertiary care hospital. The study was undertaken after the approval from Institutional ethical committee.

All clinical isolates of Candida obtained from various samples and reported as pathogenic were included. These isolates were obtained from specimens like urine, sputum, blood, pus, body fluids, vaginal swab, etc. during one calendar year (total number-100). 
Relevant clinical history of the concerned patient was noted along with HIV status and other investigations. Also repeat sample was collected. These isolates of Candida were subjected for further tests.

The isolates were first sub cultured on CHROM agar Candida plates and incubated at $37^{\circ} \mathrm{C}$ for 24 hours aerobically to check for purity and identification. All isolates were identified based on standard mycological methods, morphology on CHROM agar Candida, morphology on corn meal agar, germ tube production, sugar fermentation and sugar assimilation. All the isolates were subcultured on Sabouraud's Dextrose Agar for further tests. Following table shows the colour appearance of the different candida species on CHROM agar and their microscopic morphology on Corn Meal Agar used for identification (Patel et al 2012).

Table.1 Colony Morphology of Candida sps in Chrom agar and CMA

\begin{tabular}{|l|l|l|}
\hline Candida species & Colour on Chrom agar & Morphology on CMA \\
\hline C.albicans & Light green & Chlamydoconidia on hyphae \\
\hline C.glabrata & Pink to purple & Only blastospores \\
\hline C.tropicalis & Blue with pink halo & Multibranchedpseudohyphae \\
\hline C.parapsilosis & Cream to pale pink & Sage brush or shaggy star appearance \\
\hline C.guilleirmondii & Pink to purple & Pseudohyphae very fine and short with small cells \\
\hline C.dubliniensis & Dark green & Chlamydoconidia on hyphae \\
\hline
\end{tabular}

Antifungal susceptibility was done according to National Committee for Clinical Laboratory Standard, 2004-method for antifungal disc diffusion susceptibility for yeast with approved guidelineM44-A. Following antifungal discs were used; Fluconazole (25mcg/disc), AmphotericinB (100unit/disc), Voriconazole (1 mcg/disc), Itraconazole (10mcg/disc), Ketoconazole (50mcg/disc). The inoculum standardized to 0.5 McFarland turbidity standard. Supplemented Mueller Hinton agar (MullerHinton agar $+2 \%$ glucose $+0.5 \mu \mathrm{g} / \mathrm{ml}$ methylene blue dye) i.e. GBM medium was used for sensitivity (Choudhari et al 2009). C.albicans ATCC 10231 and C.tropicalis 66029 were used as controls in the study.

\section{Results and Discussion}

All clinical isolates of Candida were obtained from various samples such as urine, sputum, pus, pleural fluid, catheter tip, blood and throat swab. Out of 100 Candida isolates highest number of isolates i.e. $58 \%$ were obtained from urine followed by sputum $13 \%$, pus $9 \%$, pleural fluid, vaginal swab and catheter tip each $3 \%$ as well as from blood and throat swab2\% and $1 \%$ respectively (Table no. 1 ).

We have studied the association of the risk factors in all the patients from whom the Candida species were isolated. As seen in table no. 2 diabetes mellitus was found to be the most frequently associated risk factor (34\%), pregnancy $(16 \%)$ being the second followed by use of catheter (12\%), pneumonia $(11 \%)$, and surgery $5 \%$ etc. (table no.2)

100 isolates were identified by both CHROM agar and conventional methods. Out of 100 isolates $41 \%$ were C.albicans. The non albicans candida isolated were C.tropicalis (35\%), C. glabrata (14\%), C.dubliniensis (6\%), C. parapsilopsis (3\%) and C.gullermondii (1\%) identified by both CHROM agar and conventional methods (Table no.3). 
In present study $41 \%$ of isolates were C.albicans and $59 \%$ were non albicans candida (NAC). Proportion of NAC were significantly more than C.albicans. (Table no. 4)

The susceptibility pattern of Candida species to frequently used antifungal drugs was varied $82 \%$ Candida species were sensitive to Amphotericin B, $17 \%$ SDD and $1 \%$ were resistant. $85 \%$ Candida species were sensitive to Fluconazole, 4\% SDD and 11\% resistant. $88 \%$ Candida species were sensitive to Voriconazole and $12 \%$ resistant. $36 \%$ Candida species were sensitive to Ketoconazole, 38\% SDD and 26\% resistant. $45 \%$ Candida species were sensitive to Itraconazole, $11 \%$ SDD and $44 \%$ resistant.

A total of 100 Candida isolates from 99 various clinical specimens were included in our study, one specimen showed mixed growth, of which highest number of isolates 1were obtained from urine (58\%) followed by sputum (13\%) and pus (9\%) (Table-1). Patel LR (Patel, et al., 2012) also reported the highest no. of isolates $(30.5 \%)$ from urine followed by sputum (28.9\%). Pethani, et al., 2013 also reported highest number of isolates from urine and blood (30.64\%) followed by sputum (19.35\%).

We have studied the association of the predisposing factors in all the 99 patients from whom the Candida species were isolated (total 100 isolates). As seen in table no. 2 diabetes mellitus was the most frequently associated risk factor $(34.34 \%)$ in our study. Experimental evidence in vitro shows that a glucose concentration of 150 $\mathrm{mg} / 100 \mathrm{ml}$ increases the growth of Candida. This may probably hold true in the human body, that an increase in concentration of glucose in the tissue, blood \& urine promotes the growth of Candida (Dharwad et al., 2011). A comparison of the incidence of diabetes among the cases of Candidiasis is shown in following table.

\begin{tabular}{|l|l|l|}
\hline S.no. & Study & $\begin{array}{l}\text { Percentage } \\
\text { Diabetic patients }\end{array}$ \\
\hline 1 & Deorukhkar.et al & 40 \\
\hline 2 & Dharwad et al & 32 \\
\hline 3 & Kashid et al & 31.97 \\
\hline 4 & Present study & 34 \\
\hline
\end{tabular}

The findings of the present study correlated well with these studies.

In the present study, pregnancy was the second commonest predisposing factor that is $16 \%$ (table 2). The increased prevalence of genital candidiasis in pregnancy is due to the increase in the glycogen content of the vagina and thus increasing the colonization of Candida (Bankar et al., 2012). Vaginal candidiasis is extremely common infection in $60-70 \%$ women during their reproductive age at least once in their lives (Babin, et al., 2013). Amar, et al., 2013 also found pregnancy as the second most predisposing factor i.e. $22.3 \%$. Followed by Kashid, et al., (2011) who showed it to be $14.23 \%$. The findings of present study correlated well with these studies.

In our study we evaluated the usefulness of the chromogenic agar medium for identification of Candida species. We could identify all 100 Candida species on CHROM agar. The results on CHROM agar almost matched that of conventional method (table 3). CHROM agar candida, Hi media, easily and accurately identifies following Candida species namely C.albicans, C.tropicalis, $C$. glabrata, $C$. parapsilosis and $C$. guillermondii based on color and morphological features. This finding is in agreement with the previous studies done by Sanjeev Kumar et al., 2013; Amar et al., 2013 and Vijaya et al., 2011 who also reported accurate differentiation of most common species on CHROM agar namely C.albicans, C.tropicalis, C.parapsilosis and C.glabrata. 
Table.1a Sample Wise Distribution of Candida species

\begin{tabular}{|c|c|c|c|c|c|c|c|c|c|}
\hline Species & Urine & Sputum & $\begin{array}{c}\text { Pu } \\
\text { s }\end{array}$ & $\begin{array}{c}\text { Pleural } \\
\text { Fluid }\end{array}$ & $\begin{array}{c}\text { V. } \\
\text { swab }\end{array}$ & $\begin{array}{c}\text { C. } \\
\text { tip }\end{array}$ & $\begin{array}{c}\text { Blo } \\
\text { od }\end{array}$ & $\begin{array}{c}\text { Throat } \\
\text { swab }\end{array}$ & Other \\
\hline C.albicans & 26 & 6 & 2 & 1 & 2 & 2 & 1 & 1 & 0 \\
\hline C.tropicalis & 19 & 6 & 3 & 2 & 0 & 1 & 1 & 0 & 3 \\
\hline C.glabrata & 9 & 1 & 2 & 0 & 1 & 0 & 0 & 0 & 0 \\
\hline C.dublinensis & 1 & 3 & 2 & 0 & 0 & 0 & 0 & 0 & 0 \\
\hline C.parapsilois & 2 & 1 & 0 & 0 & 0 & 0 & 0 & 0 & 0 \\
\hline C.guillermondii & 1 & 0 & 0 & 0 & 0 & 0 & 0 & 0 & 0 \\
\hline Total & 58 & 13 & 9 & 3 & 3 & 3 & 2 & 1 & 3 \\
\hline
\end{tabular}

V. swab- Vaginal swab, C.tip -catheter tip

Maximum number of candida isolates were obtained from urine sample.

Table.2 Distribution of Risk Factors in patients with Candida isolate.

\begin{tabular}{|c|c|}
\hline Predisposing factor & No. of patients \\
\hline Diabetes & $34(34.34 \%)$ \\
\hline Pregnancy & $16(16.16 \%)$ \\
\hline Catheter & $12(12.12 \%$ \\
\hline Pneumonia & $11(11.11 \%)$ \\
\hline Surgery & $5(5.5 \%)$ \\
\hline Trauma & $4(4.4 \%)$ \\
\hline Tuberculosis & $3(3.3 \%)$ \\
\hline Malignancy & $2(2.2 \%)$ \\
\hline Neonatal sepsis & $2(2.2 \%)$ \\
\hline Prolonged hospital stay & $4(4.4 \%)$ \\
\hline Predisposing factor not known & $6(6.6 \%)$ \\
\hline Total & $99(100 \%)$ \\
\hline
\end{tabular}

Diabetes was found to be most common risk factor associated with candida infection.

Table.3 Species identification by CHROM agar method and conventional method

\begin{tabular}{|c|c|c|}
\hline Species & CHROM agar & Conventional \\
\hline C.albicans & 41 & 41 \\
\hline C.tropicalis & 35 & 35 \\
\hline C.glabrata & 14 & 14 \\
\hline C.dublinensis & 6 & 6 \\
\hline C.parapsilosis & 3 & 3 \\
\hline C. guillermondii & 1 & 1 \\
\hline Total & 100 & 100 \\
\hline
\end{tabular}

All the isolates were identified $100 \%$ by both methods. 
Table.4 Number of Candida albicans and non albicans candida(NAC)

\begin{tabular}{|c|c|c|}
\hline Species & Total isolates & Percentage \\
\hline C.albicans & 41 & 41 \\
\hline NAC & 59 & 59 \\
\hline
\end{tabular}

$\mathrm{Z}=2.404 ; \mathrm{P}=0.016$; Proportion of NAC were significantly more than C.albicans

Table.5 Isolated Candida species and its antifungal sensitivity pattern.

\begin{tabular}{|c|c|c|c|c|c|c|c|c|c|c|c|c|c|c|c|}
\hline \multirow[t]{2}{*}{ Species } & \multicolumn{3}{|c|}{ Amphotericin-B } & \multicolumn{3}{|c|}{ Fluconazole } & \multicolumn{3}{|c|}{ Voriconazole } & \multicolumn{3}{|c|}{ Ketoconazole } & \multicolumn{3}{|c|}{ Itraconazole } \\
\hline & $\mathrm{S}$ & SDD & $\mathrm{R}$ & $\mathrm{S}$ & SDD & $\mathrm{R}$ & $\mathrm{S}$ & SDD & $\mathrm{R}$ & $\mathrm{S}$ & SDD & $\mathrm{R}$ & $\mathrm{S}$ & SDD & $\mathrm{R}$ \\
\hline $\begin{array}{l}\text { C.albicans } \\
\text { (41) }\end{array}$ & $\begin{array}{c}40 \\
(97.56)\end{array}$ & $\begin{array}{c}0 \\
(0)\end{array}$ & $\begin{array}{c}1 \\
(2.43)\end{array}$ & $\begin{array}{c}33 \\
(80.48)\end{array}$ & $\begin{array}{c}1 \\
(2.43)\end{array}$ & $\begin{array}{c}7 \\
(17.7)\end{array}$ & $\begin{array}{c}33 \\
(80.48)\end{array}$ & $\begin{array}{c}0 \\
(0)\end{array}$ & $\begin{array}{c}8 \\
(19.51)\end{array}$ & $\begin{array}{c}18 \\
(43.9)\end{array}$ & $\begin{array}{c}12 \\
(29.26)\end{array}$ & $\begin{array}{c}11 \\
(26.82)\end{array}$ & $\begin{array}{c}20 \\
(48.78)\end{array}$ & $\begin{array}{c}8 \\
(19.51)\end{array}$ & $\begin{array}{c}13 \\
(31.70)\end{array}$ \\
\hline $\begin{array}{r}\text { NAC } \\
(59)\end{array}$ & $\begin{array}{c}42 \\
(71.18)\end{array}$ & $\begin{array}{c}17 \\
(28.81)\end{array}$ & $\begin{array}{c}0 \\
(0)\end{array}$ & $\begin{array}{c}52 \\
(88.13)\end{array}$ & $\begin{array}{c}3 \\
(5.08)\end{array}$ & $\begin{array}{c}4 \\
(6.77)\end{array}$ & $\begin{array}{c}55 \\
(93.52)\end{array}$ & $\begin{array}{c}0 \\
(0)\end{array}$ & $\begin{array}{c}4 \\
(6.77)\end{array}$ & $\begin{array}{c}18 \\
(30.5)\end{array}$ & $\begin{array}{c}26 \\
(44.06)\end{array}$ & $\begin{array}{c}15 \\
(25.42)\end{array}$ & $\begin{array}{c}25 \\
(42.37)\end{array}$ & $\begin{array}{c}3 \\
(5.08)\end{array}$ & $\begin{array}{c}31 \\
(52.54)\end{array}$ \\
\hline $\begin{array}{l}\text { Total } \\
(100)\end{array}$ & $\begin{array}{c}82 \\
(82 \%)\end{array}$ & $\begin{array}{c}17 \\
(17 \%)\end{array}$ & $\begin{array}{c}1 \\
(1 \%)\end{array}$ & $\begin{array}{c}85 \\
(85 \%)\end{array}$ & $\begin{array}{c}4 \\
(4 \%)\end{array}$ & $\begin{array}{c}11 \\
(11 \%)\end{array}$ & $\begin{array}{c}88 \\
(88 \%)\end{array}$ & $\begin{array}{c}0 \\
(0)\end{array}$ & $\begin{array}{c}12 \\
(12 \%)\end{array}$ & $\begin{array}{c}36 \\
(36 \%)\end{array}$ & $\begin{array}{c}38 \\
(38 \%)\end{array}$ & $\begin{array}{c}26 \\
(26 \%)\end{array}$ & $\begin{array}{c}45 \\
(45 \%)\end{array}$ & $\begin{array}{c}11 \\
(11 \%)\end{array}$ & $\begin{array}{c}44 \\
(44 \%)\end{array}$ \\
\hline
\end{tabular}

S-Susceptible, SDD-Susceptible dose dependent, R-Resistant 
Fig.1 Gram's staining showing gram positive budding yeast cells at $100 \mathrm{x}$

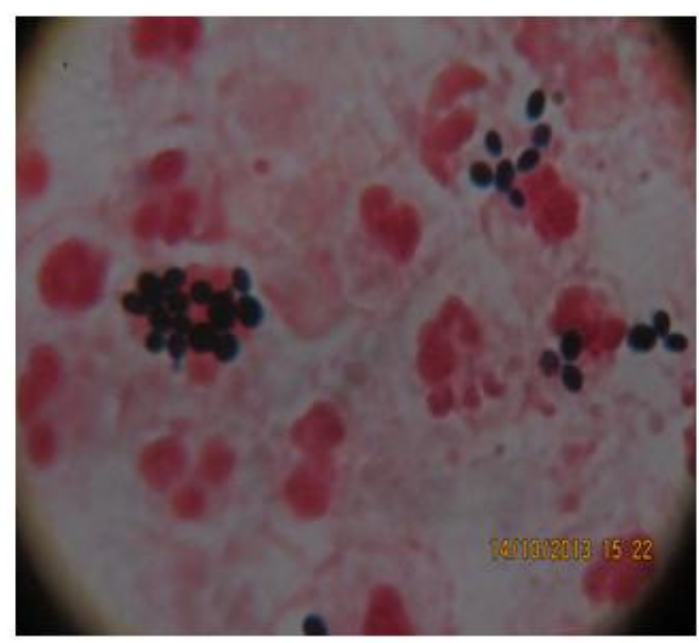

Fig.2 Candida tropicalis on CHROM agar

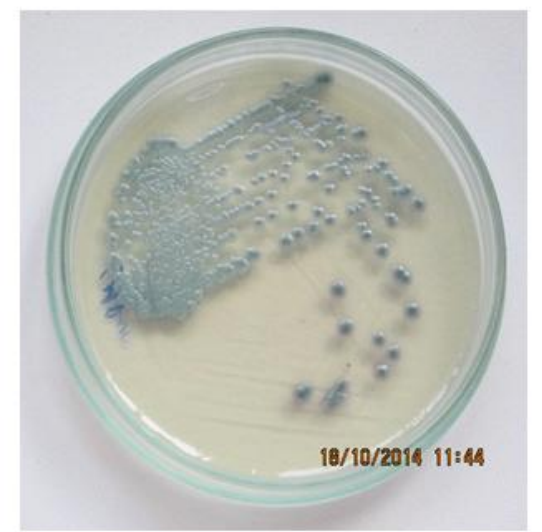

Fig.3 Microscopic appearance of Candida tropicalis on CMA at $40 \mathrm{X}$

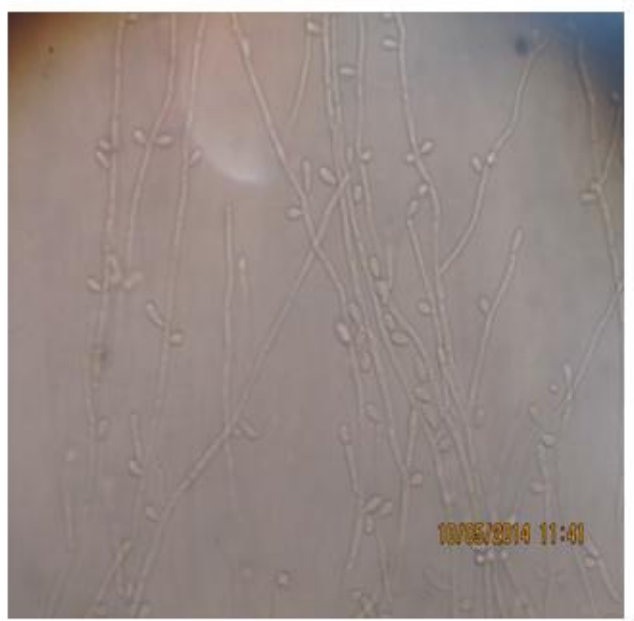


Fig.4 Candida albicans on CHROM agar .

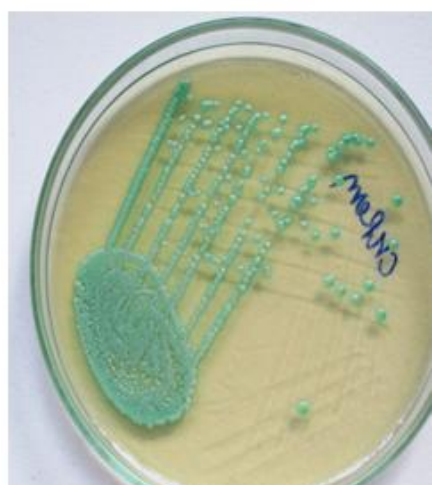

Fig.5 Microscopic appearance of Candida albicans on CMA at $40 \mathrm{X}$

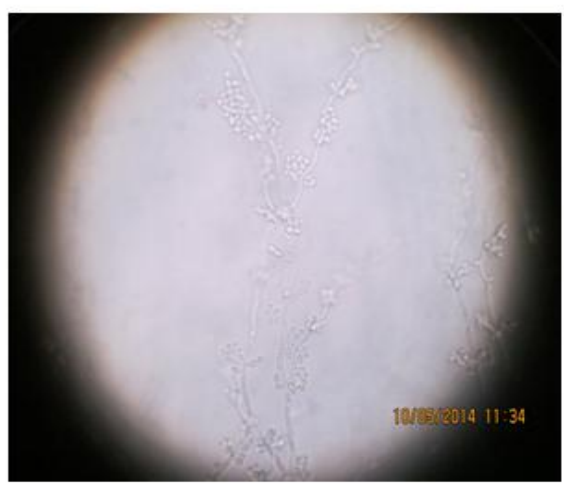

Fig.6 Candida glabrata on CHROM agar.

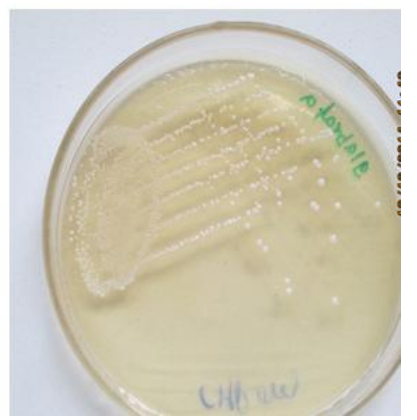

Fig.7 Microscopic appearance of Candida glabrata on CMA at $40 \mathrm{X}$

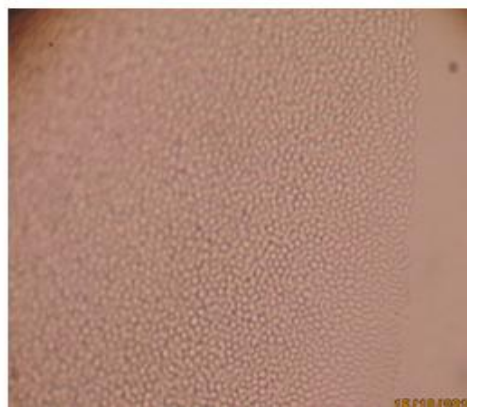

Fig.8 Candida parapsilosis on CHROM agar 


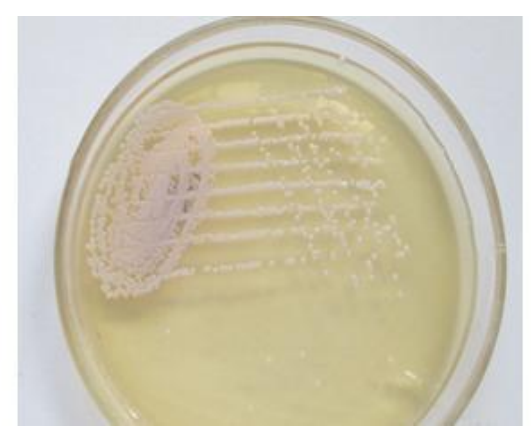

Fig.9 Microscopic appearance of Candida parapsilosis on CMA at $40 \mathrm{X}$

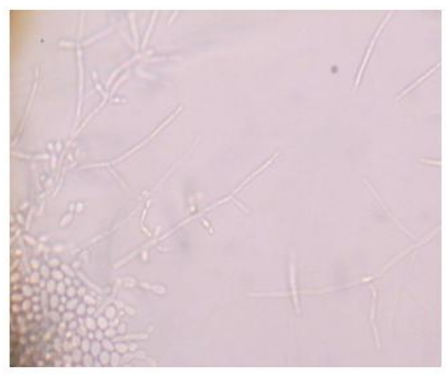

Fig.10 Anti-fungal sensitivity test by disk diffusion method

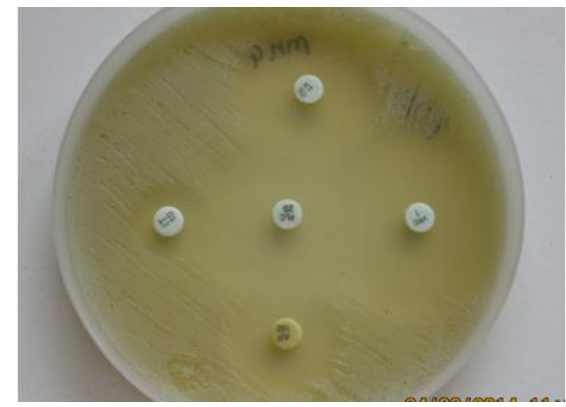

The result of present study suggests that CHROM agar medium helps to rapidly identify clinically important Candida species while potentially decreasing laboratory cost.

Studies over the years have shown that there is a considerable increase in the NAC. In our study we have found $41 \%$ of C.albicans and $59 \%$ of NAC isolates (table 4). This was in agreement with the findings of the studies by Sanjeev Kumar et al. (Kumar S, et al., 2013) showing $60 \%$ NAC \& $40 \%$ C.albicans isolation. Pethani et al., 2013 showed $59 \%$ NAC and $41 \%$ C.albicans. Anaparthy, et al., 2011 isolated 60.9\% NAC and $39.1 \%$ C.albicans. The predominant NAC isolate observed in our tertiary care center was C.tropicalis. This was in agreement with the studies conducted by Sanjeev Kumar et al (Kumar S et al., 2013), Anaparthy U (AnaparthyU et al., 2011) and ManjunathVeenaet al., (Manjunath V et al., 2012). During recent decades, several countries around the world have witnessed a change in the epidemiology of Candida infections, characterized by a progressive shift from a predominance of Candida albicans to non albicans candida species (including Candida glabrata) one of the reasons being the use of prophylactic 
antifungal agents.

The susceptibility pattern of Candida spp. to frequently used antifungal drugs was varied. $82 \%$ Candida spp. were sensitive to Amphotericin-B, 17\% SDD and 1\% were resistant (table 5). ChangdevAher et al (Aher C et al., 2014) stated that $85.1 \%$ Candida spp. were sensitive to Amphotericin-B \& 14.9\% were resistant. Kashid R A et al., (Kashid R A et al., 2011) showed that $98.63 \%$ Candida spp. were sensitive to Amphotericin-B.

In the present study 36\% Candida spp. were sensitive to Ketoconazole where as $38 \%$ SSD and $26 \%$ species were resistant to Ketoconazole (table-5). Abdulha et al., (Abdulha et al., 2014) detected $42.9 \%$ sensitive, 29.6\% SDD \&27.6\% resistant strains to Ketoconazole.

In present study $88 \%$ Candida spp. were sensitive to Voriconazole \& remaining are resistant (table 5). While Dharwad S. et al., (Dharwad S. et al., 2011) stated that $66 \%$ Candida species were sensitive, 24\% SDD\& $16 \%$ were resistant to Voriconazole.

In present study Itraconazole showed $45 \%$ sensitivity, $11 \%$ SDD, \& $44 \%$ resistance (table 5). Dharwad, et al., 2011) in their study showed that $48 \%$ Candida spp. were sensitive to Itraconazole $32 \%$ SDD \& $20 \%$ were resistant.

According to our study, 85\% Candida spp. were sensitive, 4\% SDDand11\% were resistant to Fluconazole (table 5), while Aher, et al., 2014 stated $60.9 \%$ sensitivity\& $39.1 \%$ resistance to Fluconazole. Kashid R et al., 2011 showed that 59.18\% species were sensitive, $10.20 \%$ SDD \& $30.61 \%$ resistance to Fluconazole. It shows that different patterns of sensitivity to Fluconazole is observed in different regions or hospitals depending upon the epidemiological conditions.

Amphotericin shows good sensitivity against all the candidial species in vivo. So it still remains a good choice in case the facility for antifungal sensitivity is not available.

Rapid identification of different candida species is possible by growth on CHROM agar within 48 hours while conventional methods takes longer period. We have found variety of candida species in the study which are less common and may pose a further, future threat to optimal antifungal therapy and underlines the importance of accurate species identification and antifungal sensitivity testing, as susceptibility varies according to the type of infecting candida spp. Hence use of CHROM agar with disc diffusion susceptibility testing on GMB agar is a simple, rapid and cost effective method that can be used in resource restricted settings without requirement of any costly instrument. It also plays a major role in tracking the development of antifungal resistance in epidemiological studies.

\section{Acknowledgment}

We are thankful to Mrs. Alka Gore, Statistician, Department of PSM, BVDUMCH, Sangli for her help in Statistical analysis.

\section{Reference}

Aher, C.S. 2014. Species distribution, virulence factors and Antifungal Susceptibility profile of Candida isolated from Oropharyngeal lesion of HIV infected patients. Int. J. Curr. Microbiol. Appl. Sci., 3(1): 453-460.

Amar, C.S. et al. 2013. Study of Prevalence And Antifungal Susceptibility Of Candida. Int. J. Pharma. Bio. Sci., 4(2): 361-81.

Anaparthy, U., et al. 2011. Isolation and Characterisation of Candida species from Oropharyngeal Secretion of HIV positive 
individuals. Dermatol. Online, 2(3): 119124.

Babin, D., Kotigadde, S., Rao, P.S., Rao, T.V. 2013. Clinico-mycological profile of vaginal candidiasis in a tertiary care hospital in Kerala. Int. J. Res. Biol. Sci.,. 3(1): 55-59.

Bankar, S.M., Powar, R.M., Patil, S.A., Kalthur, S.G. 2012. Prevalence of non-albicans candida infection in Maharashtrian women with leucorrhea. Ann. Trop. Med. Public Health, 5(2): 119-123.

Charlene, S.D., W. Heneine, Topley. Wilson's Mycology Vol- , $10^{\text {th }}$ ED, Chapter 30: 579624.

Chaudhary, U., Deep, A., Chabbra, N. 2009. Rapid Identification and antifungal susceptibility pattern of Candida isolates from critically III patients with candiduria. J. Infect. Dis. Antimicrob. Agents, 26(2): 50-53.

Deorukhkar, S., Saini, S. 2012. Species Distribution And Antifungal Susceptibility Profile Of Candida species Isolated From Blood Stream Infection. J. Evol. Med. Dent. Sci.,.1(3): 241-49.

Dharwad, S., Saldanha, R.M. 2011. Species identification of Candida isolates in various clinical specimens with their antifungal susceptibility patterns. J. Clin. Diag. Res., 5(6): 1177-81.

Enoch, D.A., Ludlam, H.A., Brown, N.M. 2006. Invasive fungal infections: a review of epidemiology and management options. $J$. Med. Microbiol., 55: 809-18.

Jagdish Chander. 2009. editor Text book of Medical Mycology $3^{\text {rd }}$ edition chapter 20: 266-90.

Kashid, R.A., Belawadi, S., et al. 2011. Characterization and antifungal susceptibility testing for candida species in a tertiary care hospital. J. Health Sci. Res., 2(2): 1-7.
Kothavade, R.J., Kura, M.M., Valand, A.G., Panthaki, M. 2011. Candida tropicalis: its prevalence, pathogenicity and increasing resistance to fluconazole. J. Med. Microbiol., 59: 873-80.

Kumar, S., Vyas, A., Kumar, M., Mehra, S.K. 2013. Application of CHROM agar Candida for Identification of Clinically Important Candida species and their Antifungal Susceptibility Pattern. Int. J. Med. Res., 4(4): 3600-6.

Manjunath, V., Vidya, G.S., Sharma, A. et al. 2012. Speciation of Candida by Hicrome agar and Sugar assimilation test in both HIV infected and non-infected patients. Int. J. Biol. Med. Res., 3(2): 1778-82.

Pfaller, M.A., Diekema, D.J., Gibbs, D.L. et al. 2010. J. Clin. Microbiol., 48(4): 366-377.

Saravana Bhavan, P. 2010. Culture and Identification of Candida albicans from Vaginal Ulcer and Separation of Enolase on SDS-PAGE. Int. J. Biol., 2(1): 84-90.

Patel, L.R., Pethani, J.D., Bhatia, P., Rathod, S.D., Shah, P.D. 2012. Prevalence of Candida Infection And Its Antifungal Susceptibility Pattern In Tertiary Care Hospital, Ahemedabad. Nat. J. Med. Res., 2(4): 439-41.

Pethani, J.D., Gusani, J., Rathod, S., Shah, P.D. 2013. Candida species Identification from Clinical Specimens and Its Changing Pattern In A Tertiary Care Hospital. Indian J. Appl. Basic Sci., 15(20): 17-22.

Price, M.F., LaRocco, M.T., Gentry, L.O. 1994. Fluconazole susceptibility of Candida species and distribution of species recovered from blood cultures over a 5 year period. Antimicrob. Agents Chemother., 38(6): 1422-24.

Vijaya, D., Harsha, T.R., Nagaratnamma, T. 2011. Candida Speciation Using Chrom Agar. J. Clin. Diag. Res., 5(4): 755-5.

\section{How to cite this article:}

Shilpa Rajesh Shah and Swati Raghunath Sapkal. 2016. Study of Risk Factors Associated with Candidiasis and Identification and Antifungal Susceptibility Pattern of Candida Isolates in a Tertiary Care Hospital. Int.J.Curr.Microbiol.App.Sci. 5(6): 55-65. doi: http://dx.doi.org/10.20546/ijcmas.2016.506.007 